\title{
ENHANCING THE MESSAGES DISPLAYED ON DYNAMIC MESSAGE SIGNS
}

\author{
Chun-Ming Yang ${ }^{1}$, Dusty Waters ${ }^{2}$, Carmeris C. Cabrera ${ }^{1}$, \\ Jyh-Hone Wang ${ }^{1}$, Charles E. Collyer ${ }^{2}$ \\ ${ }^{1}$ Department of Industrial and Manufacturing Engineering \\ ${ }^{2}$ Department of Psychology \\ University of Rhode Island \\ Kingston, Rhode Island, USA \\ E-mail: jhwang@egr.uri.edu
}

\begin{abstract}
Summary: A human factors study was carried out to help enhance ways to communicate with highway motorists through dynamic message signs (DMS). Overhead mounted DMSs have been increasingly used by highway authorities in the United States to present real-time traffic information and travel advice to motorists. It is critical to post sign messages that can be quickly and clearly understood by motorists, especially in high-volume traffic and construction/repair zones. Properly worded and formatted sign messages could spell the difference between comprehension and confusion. Message display factors investigated in the study include display effects, color schemes, wording, and formats. Two approaches were employed in this study. First, a questionnaire survey was developed to collect motorists' preferences regarding various message display factors. Second, a series of lab driving simulation experiments were set up to assess the effects of these factors and their interactions on motorists' comprehension of DMS messages. Study results suggested that static, one-framed messages with more specific wording and no abbreviations were preferred. Amber or green or a green-amber combination were the most favored colors. Younger subjects took less response time to the DMS stimuli with higher accuracy than older subjects. There were no significant gender differences.
\end{abstract}

\section{INTRODUCTION}

The use of dynamic message signs (DMS) as part of highway management systems has become popular in the United States in recent years. DMS serves as a major communication device between motorists and highway authorities to promote safe and efficient driving on highways. In addition, it plays an important role in the Intelligent Transportation System (ITS) by displaying real-time information and advice about roadway and traffic conditions. Thus, a properly designed and displayed message on DMS is especially important to a motorist who has to detect, comprehend, and act on signage information while being occupied with the driving task. The selection of words and their formats are critical since poorly worded and/or formatted messages could confuse drivers and cause safety concerns. An extensive review of past studies regarding DMS was carried out. Important findings from this review are summarized below.

A preference survey conducted by Wardman, Bonsall, \& Shires (1997) concluded that the impact of DMS information depended on the message content, local circumstances and motorists' characteristics. Another survey (Lai and Wong, 1998) found that driver preference could be affected by presenting identical traffic information in different formats. Two similar DMS studies of motorists' understanding of abbreviations were conducted in Texas and New Jersey (Durkop and Dudek, 2001; Hustad and Dudek, 1999) and identified 24 abbreviations that were 
understood at an acceptable level, but regional differences with respect to understanding of the abbreviations were noted. Dudek and Ullman (2002) investigated issues relevant to the use of the dynamic characteristics of DMS and suggested: (i) one-frame DMS messages should not be flashed, (ii) a line on a one-frame DMS message should not be flashed, and (iii) a line on a twoframe DMS message should not be alternated while keeping other lines the same. Guerrier and Wachtel (2001) used a low-cost, interactive driving simulator to study driver response to variable message signs of differing message length and format. Results showed consistent and significant age effects across all tested conditions. In addition, all drivers responses were significantly poorer under the two-phase DMS. Wang and Cao (2003; 2005) employed a video-based simulation approach to study subjects' response to a variety of VMS stimulus messages. Results indicated that static, one-frame messages took less response time than sequential, two-frame ones; messages with fewer lines were responded to faster in both static and sequential ones. Older subjects required a longer response time with less accuracy.

The above mentioned studies investigated various aspects of message display on DMS and resulted in many significant findings. Since the advancement in DMS technology has made it possible to display messages with multiple colors and various formats, there is a need to study motorists' comprehension and response to these recent DMS features. A human factors study was designed and carried out to examine how various features in DMS messaging affected a motorist's understanding of the message in terms of response time and accuracy. The factors examined here include: color scheme, number of message frames, display effect, wording and format. Demographic difference in motorists' age, gender and native language were also taken into account in the study. Special attention was placed on the elder population and non-English population since they might find sign reading challenging during driving.

\section{DESCRIPTION OF THE STUDY}

This study took two approaches to evaluate the effects of various DMS messaging factors and their combinations on motorists, questionnaire surveys and lab simulation. The first collected respondents' opinions and preferences through a number of multiple-choice questions via a PowerPoint presentation, while the second tested motorists' response to a series of digitally generated DMS stimuli in a simulated driving experiment. Both parts of the study took place in the Motorist Performance Laboratory at the University of Rhode Island.

\section{Subjects}

Subjects with valid driver licenses and driving experience on interstate highways, with normal or near-normal eyesight, and possessing certain demographic characteristics were recruited to participate in the research. They represented motorists from three age groups: 20-40 years old, 41-60 years old and 61 and above. Among each group there were equal numbers of male and female participants. These participants possessed different linguistic backgrounds; some were native English speakers while others were not. Thirty-six subjects participated in the study. Each subject was asked to sign a consent form and fill out a demographic questionnaire prior to starting the survey and driving simulation. Additionally, subjects were briefed and instructed about the purpose and procedures. Subjects were paid for their time to encourage their participation in the study. 


\section{Questionnaire Survey}

There were a total of forty-four multiple choice questions where DMS messages with various features were displayed on Microsoft PowerPoint ${ }^{\circledR}$. Each slide contained questions made up of three to four choices. The message content for the signs was selected from a library of messages provided by the Rhode Island Department of Transportation (RIDOT). They dealt with common situations confronted by motorists when driving on the highway such as: accidents, congestion, construction, or icy roads. Every sign utilized a standard black background and the questions evaluated several messaging factors and their combinations. The factors and their respective levels assessed in the survey were: frame scheme (one-frame and two-frame), flashing effect (one-line flashing, all lines flashing and no lines flashing), color scheme (red, green, and amber), color combinations (green \& amber, red \& amber, and tri-color) and wording scheme (less specific, more specific and very specific). Different choices in each question used the same message content, but were displayed with different effects with respect to a factor. Each question investigated only the effects of one factor at a time. Questions that examined a specific factor preference were replicated four times with different messages each time to assure consistency. All questions were randomly distributed throughout the survey.

\section{Driving Simulation Experiment}

Experimental Design. A series of full factorial experiments were employed to administer the lab driving simulation tests with two groups of factors: within-subject factors and between-subject factors (Table 1). Within-subject factors included: display effect and color scheme. Betweensubject factors were age and gender. With two replicates, each subject went through 96 trials in the experiment. The 96 trials were consisted of two replications of 3 messages, each with 3 display effects and 4 color schemes, plus 12 fake messages.

Table 1. Experiment factors and their levels

\begin{tabular}{|c|c|}
\hline Within-subject Factors & Level \\
\hline Display effect (D) & Still (0), Frame flashing (1), Line flashing (2) \\
\hline Color scheme (C) & Amber (1), Green \& amber (2), Red \& amber (3), Tricolor (4) \\
\hline Between-subject Factors & $20 \sim 40(1), 41 \sim 60$ (2), Above 60 (3) years old \\
\hline Subject's age (A) & Female (1), Male (2) \\
\hline Subject's gender (G) & \\
\hline
\end{tabular}

The statistical model employed in the study was:

$$
\mathrm{T}=\mu+\mathrm{D}_{\mathrm{i}}+\mathrm{C}_{\mathrm{j}}+(\mathrm{DC})_{\mathrm{ij}}+\mathrm{A}_{\mathrm{k}}+\mathrm{G}_{1}+(\mathrm{AG})_{\mathrm{kl}}+\varepsilon_{\mathrm{ijkl}}
$$

where $\mathrm{T}$ is subject's response time (in seconds), $\mu$ is the overall mean, and $\varepsilon$ is the error term.

Experiment Setup. A video-based driving simulation apparatus was devised to capture subjects' responses to different DMS message stimuli in a virtual driving environment. The main elements of the apparatus in the Motorist Performance Lab include a four-door 1998 Ford Taurus sedan, a Dell Dimension 4500 desktop computer with enhanced video processor, a Microsoft Sidewinder force feedback wheel, a BenQ DLP digital projector (1024 x 768 XGA resolution, 2500 ANSI 
Lumens), and a wide screen (3.67 $\mathrm{m}$ wide x $2.15 \mathrm{~m}$ high) with adjustable stands. The distance between the screen and the subject, who sat in the driver's seat of the car, was 2.24 meters. In the simulation experiment, digitally created DMS stimuli were superimposed onto a clip of real driving video and were projected onto the wide screen in front of the test vehicle. The DMS stimulus would initially appear at the far end of the video as a small dot and gradually increase in size as seen in actual driving. The stimuli were introduced in a random but controlled manner by a Microsoft Visual Basic computer program. The Sidewinder, replacing the steering wheel, was used to capture subjects' responses throughout the experiments.

Video Stimuli. The driving video was taken on Route 4 southbound between exit 5 and exit 6 in Rhode Island (RI) in March, 2004. While driving at $50 \mathrm{mph}$ under cruise control, a Canon XL1 digital video camcorder, mounted on a tripod inside a 2001 Chrysler Voyager and leveled at driver's eye height, was used to take the video. The digital video was downloaded onto a desktop computer where individual frames were extracted by Sonic Foundry VideoFactory ${ }^{\mathrm{TM}}$. The DMS stimuli were first created by Vanguard ${ }^{\circledR}$ VMS Central Controller, which was also used by RIDOT to generate and control DMS messages in RI. Each DMS stimulus was then copied, pasted, and resized to fit the DMS board in each frame of the video by Microsoft Paint ${ }^{\mathbb{R}}$. The individual frames with DMS stimuli were then rendered into a video clip. Each video clip lasted 27 seconds and was in NTSC DV avi format with $720 \times 480$ pixel resolution and 29.970 fps.

\section{Study Protocols}

The protocol for conducting the survey was as follows. Forty-four questions were presented to each subject via PowerPoint. For each question, the subject was asked to choose a DMS displaying from a few alternatives that she/he preferred if encountered while driving on a highway. Survey questions were presented sequentially and each subject was expected to mark their choice on an answer sheet. As for the driving simulation experiment, a subject, sitting in the driver's seat of the test vehicle, was given a small number of practice runs to familiarize herself/himself with the experiment before the actual one. The subject was asked to press one of the four predefined buttons in the Sidewinder to respond to the video stimuli he/she perceived. The subject's response time and accuracy for each DMS stimulus was recorded by a computer in a database. Response time was measured as the time difference between the introduction of a new stimulus and the subject's response to that stimulus, while accuracy was the ratio of the correct responses made and the total number of stimuli.

\section{RESULTS AND DISCUSSION}

\section{Questionnaire Surveys}

The questionnaire results were analyzed against six DMS messaging features: frame scheme, flashing effect, color combinations, color scheme, wording and abbreviation. For each feature, preference statistics were collected by subject's demographics. The results expressed in percentages are shown in Table 2. A number of statistical tests were conducted to investigate between-subject differences and within-subject differences.

Gender Effect. Regarding the number of frames in a DMS message, males strongly preferred one frame over two frames $(\mathrm{p}=.033)$, while females did not exhibit a significant preference. The majority of subjects felt that they were capable of reading and understanding a message on one 
frame and found it easier than reading parts from two frames. Regarding the flashing effect, both males and females preferred a DMS message with no flashing effect. This could be an indication of how flashing effects may interfere with people's comprehension of a message. Concerning the color combination, both males and females strongly favored a green-amber combination over others. If only one color was used, amber or green were much preferred over red. With respect to wording, both genders significantly preferred DMS messages with specific wordings rather than less specific ones. There was also a disinclination towards abbreviated messages for both genders. It should be noted that all subjects overwhelmingly preferred DMS messages be given as explicitly as possible; however, this means that longer reading and comprehension time would be required and could make the use of these messages impractical.

Table 2. Motorists' preferences on DMS features by demographics

\begin{tabular}{|c|c|c|c|c|c|c|c|c|}
\hline & \multicolumn{2}{|c|}{ Gender } & \multicolumn{3}{|c|}{ Age (yrs) } & \multicolumn{2}{|c|}{ Language } \\
\hline & & Male & Female & $20-40$ & $41-60$ & 61-above & English & Non-English \\
\hline \multirow{2}{*}{ 焉 昌 } & One Frame & $73.61 \%$ & $56.94 \%$ & $50.00 \%$ & $72.92 \%$ & $72.92 \%$ & $69.23 \%$ & $55.00 \%$ \\
\hline & Two Frame & $26.39 \%$ & $43.06 \%$ & $50.00 \%$ & $27.08 \%$ & $27.08 \%$ & $30.77 \%$ & $45.00 \%$ \\
\hline \multirow{3}{*}{$\frac{d}{\frac{1}{0}}}$. & Still & $52.78 \%$ & $61.11 \%$ & $41.67 \%$ & $66.67 \%$ & $66.67 \%$ & $58.65 \%$ & $45.00 \%$ \\
\hline & All Flashing & $13.89 \%$ & $4.17 \%$ & $12.50 \%$ & $4.17 \%$ & $6.25 \%$ & $5.77 \%$ & $20.00 \%$ \\
\hline & One Line Flash & $33.33 \%$ & $34.72 \%$ & $45.83 \%$ & $29.17 \%$ & $27.08 \%$ & $35.58 \%$ & $30.00 \%$ \\
\hline \multirow{3}{*}{ 흥 } & Green \& Amber & $33.33 \%$ & $44.44 \%$ & $37.50 \%$ & $39.58 \%$ & $39.58 \%$ & $33.65 \%$ & $52.50 \%$ \\
\hline & Red \& Amber & $5.56 \%$ & $8.33 \%$ & $6.25 \%$ & $8.33 \%$ & $6.25 \%$ & $6.73 \%$ & $7.50 \%$ \\
\hline & Tricolor & $23.61 \%$ & $16.67 \%$ & $29.17 \%$ & $4.17 \%$ & $27.08 \%$ & $17.31 \%$ & $27.50 \%$ \\
\hline \multirow{3}{*}{$\begin{array}{l}\text { 믕 } \\
\text { in }\end{array}$} & Amber & $52.78 \%$ & $55.56 \%$ & $56.25 \%$ & $70.83 \%$ & $35.42 \%$ & $57.69 \%$ & $45.00 \%$ \\
\hline & Green & $44.44 \%$ & $41.67 \%$ & $39.58 \%$ & $27.08 \%$ & $62.50 \%$ & $40.38 \%$ & $50.00 \%$ \\
\hline & Red & $2.78 \%$ & $2.78 \%$ & $4.17 \%$ & $2.08 \%$ & $2.08 \%$ & $1.92 \%$ & $5.00 \%$ \\
\hline \multirow{3}{*}{ 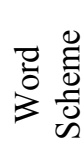 } & Less Specific & $19.44 \%$ & $20.83 \%$ & $22.92 \%$ & $10.42 \%$ & $27.08 \%$ & $22.12 \%$ & $15.00 \%$ \\
\hline & More Specific & $33.33 \%$ & $33.33 \%$ & $29.17 \%$ & $33.33 \%$ & $37.50 \%$ & $32.69 \%$ & $35.00 \%$ \\
\hline & Very Specific & $47.22 \%$ & $45.83 \%$ & $47.92 \%$ & $56.25 \%$ & $35.42 \%$ & $45.19 \%$ & $50.00 \%$ \\
\hline \multirow{4}{*}{ 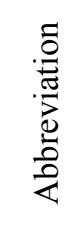 } & Not & $93.06 \%$ & $88.89 \%$ & $89.58 \%$ & $93.75 \%$ & $89.58 \%$ & $89.42 \%$ & $95.00 \%$ \\
\hline & Less & $4.17 \%$ & $4.17 \%$ & $4.17 \%$ & $4.17 \%$ & $4.17 \%$ & $4.81 \%$ & $2.50 \%$ \\
\hline & More & $1.39 \%$ & $5.56 \%$ & $4.17 \%$ & $2.08 \%$ & $4.17 \%$ & $4.81 \%$ & $0.00 \%$ \\
\hline & Very & $1.39 \%$ & $1.39 \%$ & $2.08 \%$ & $0.00 \%$ & $2.08 \%$ & $0.96 \%$ & $2.50 \%$ \\
\hline
\end{tabular}

Age Effect. According to survey results, subjects over 40 years old significantly favored oneframe DMSs over two-frame ones. With respect to the flashing effects, subjects over 40 strongly preferred still signs over flashing ones. Regarding color combination, most subjects preferred the green and amber combination. If only one color was used, red was the least preferred one. Regarding wording and abbreviations, all age groups preferred a more specific message with no abbreviations.

Native Language Effect. In order to determine if various DMS messaging features would cause preference differences between subjects with different native languages, survey results were 
compared across two groups of subjects, English vs. Non-English. The Non-English group consisted of ten subjects while the English group consisted of 26 subjects. As indicated in Table 2 , both groups preferred the one frame message, no flashing, green and amber in color, very specific wording and no abbreviations. This result may seem counterintuitive as one purpose of this research is to surmise ways to assist non-English speaking people with the understanding and perception of English DMS. Indeed, one could presume that attempting to read important messages, such as those displayed on DMS, in a language other than one's mother tongue could be a challenging task. The survey results did indicate that the non-English group had a stronger preference for very specific messages than the English group. It further indicated that the nonEnglish group was strongly against the use of abbreviated words in DMS messages.

\section{Driving Simulation Experiment}

The data collected from the driving simulation experiments were analyzed by Minitab. Analysis of variance (ANOVA) was conducted with results reported in Table 3. Color scheme, display effect and driver's age were significant at the 0.05 significance level. The interaction between color scheme and display effect and the interaction between age and gender were also significant. Main effect plots and interaction plots are shown in Figures 1 and 2.

Table 3. ANOVA results of response time

\begin{tabular}{lrrrrrr}
\hline Source & DF & \multicolumn{1}{c}{ Seq. SS } & Adj. SS & Adj. MS & \multicolumn{1}{c}{ F } & P \\
\hline Color Scheme & 3 & 2304.80 & 2334.45 & 778.15 & 273.55 & $0.000^{*}$ \\
Flash Effect & 2 & 118.02 & 133.16 & 66.58 & 23.41 & $0.000^{*}$ \\
Color Sc×Flash Ef & 6 & 55.41 & 55.41 & 9.23 & 3.25 & $0.003^{*}$ \\
Age & 2 & 1799.19 & 1819.98 & 909.99 & 319.90 & $0.000^{*}$ \\
Gender & 1 & 2.69 & 2.66 & 2.66 & 0.93 & 0.334 \\
Age×Gender & 2 & 457.71 & 462.11 & 231.06 & 81.23 & $0.000^{*}$ \\
Error & 3207 & 9122.73 & 9122.73 & 2.84 & & \\
Total & 3223 & 13860.54 & & & & \\
\hline
\end{tabular}

* Significance level $=0.05$

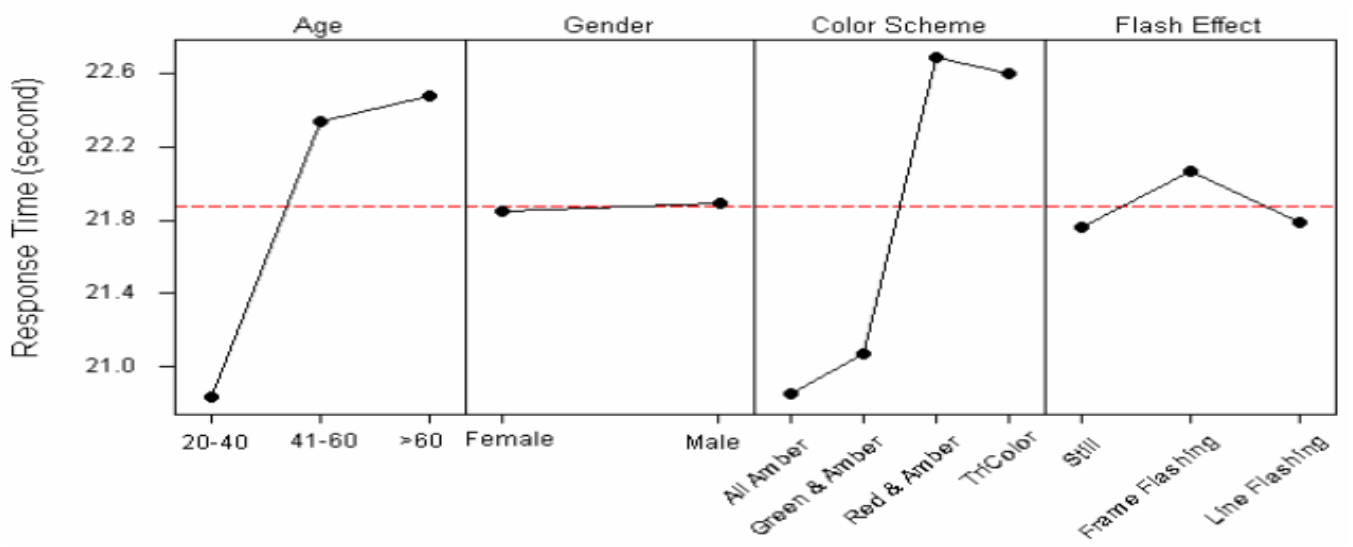

Figure 1. Main effects plots 

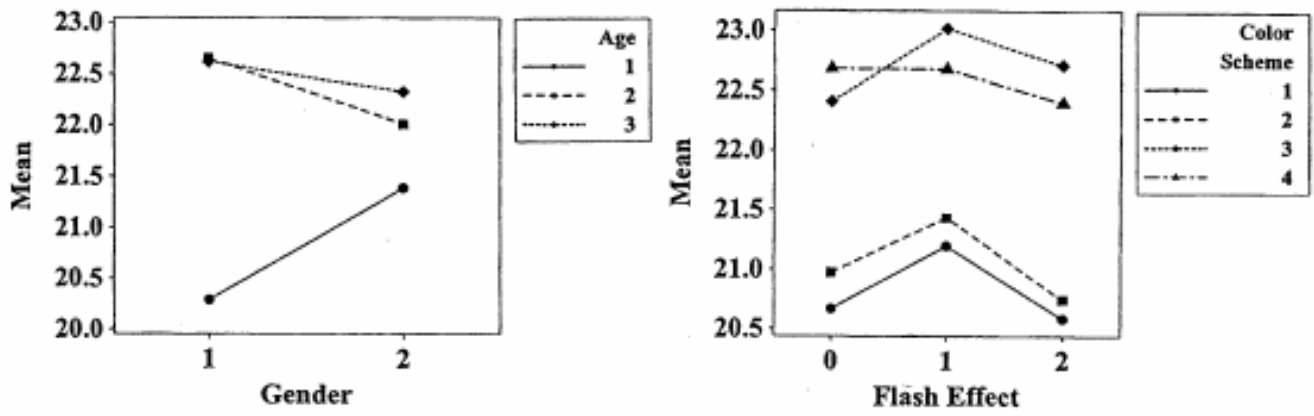

Figure 2. Interaction plots

Among the four color schemes examined, amber-colored messages resulted in the shortest response time. Subjects took longer to respond to messages containing red. As to flashing effects, subjects responded faster to still or one-line flashing messages than all-frame flashing messages. According to the interaction plots, the best display combination of a DMS message could be a still or one-line flashing message in amber color. Younger subjects responded faster with higher accuracy than older subjects. Female and male subjects did not exhibit a significant difference but female subjects responded slightly faster with higher accuracy. In addition, younger female subjects responded much faster than others, as indicated in the interaction plots.

\section{CONCLUSIONS}

A human factors study was carried out to assess motorists' responses and preferences to various combinations of DMS messaging features. Both survey and simulation results suggested that DMS signs post more specific, unabbreviated messages with still, one-framed text in amber or green color or a green-amber combination. Messages without flashing are recommended. If a flashing effect is necessary, only the first line could be flashed. Although survey results indicated certain preferences from all subjects, more studies are needed to examine these demographic differences. Overall, the present findings suggest a specific set of DMS features that might help traffic engineers and highway management design driver-friendly DMS signs that could be noticed, understood and responded to in a more timely fashion. Safer and more proactive driving experiences could then be achieved by applying these DMS signs.

\section{ACKNOWLEDGMENTS}

The authors wish to thank the Rhode Island Department of Transportation and the University of Rhode Island Transportation Center for their support and guidance in the execution of this project.

\section{REFERENCES}

Dudek, C.L. and Ullman, G.L. (2002). Flashing Messages, Flashing Lines, and Alternating One Line on Changeable Message Signs. Transportation Research Record, 1803, 94-101.

Durkop, B.R., and Dudek, C.L. (2001). Texas Driver Understanding of Abbreviations for Changeable Message Signs. Transportation Research Record, 1748, 87-95. 
Guerrier, J.H., Wachtel, J.A. (2001). A Simulator Study of Driver Response to Changeable Message Signs of Differing Message Length And Format (abstract). Proceedings of the First International Driving Symposium on Human Factors in Driver Assessment, Training and Vehicle Design, Aspen, Colorado, 164-165.

Hustad, M.W., Dudek, C.L. (1999). Driver Understanding of Abbreviations on Changeable Message Signs in New Jersey. Transportation Research Record, 1689, 46-52.

Lai, K.H., Wong, W.G. (1998). The Influence of Traffic Information Presentation Format on Driver Behavior. Proceedings of the Third Hong Kong Society for Transportation Studies, 353-358.

Wardman, M., Bonsall, P.W., Shires, J.D. (1997). Motorist Response to Variable Message Signs: A Stated Preference Investigation. Transportation Research $-C$, 5 (6), 389-405.

Wang, J.H., and Cao, Y. (2003). A Human Factors Study on Message Design of Variable Message Sign. International Journal of Industrial Engineering, Vol. 10, No. 4, 339-344.

Wang, J.H., and Cao, Y. (2005). Assessing the Design and Display of Messages on Portable Variable Message Signs. Proceedings of the $84^{\text {th }}$ Annual Meeting of Transportation Research Board, CD-ROM, paper no. 05-2186, 1-23. 